PUPT-1567

\title{
Halpern-Huang directions in effective scalar field theory
}

\author{
Vipul Periwal ${ }^{\dagger}$ \\ Department of Physics \\ Princeton University \\ Princeton, New Jersey 08544-0708
}

Halpern and Huang recently showed that there are relevant directions in the space of interactions at the Gaussian fixed point. I show that their result can be derived from Polchinski's form of the Wilson renormalization group. The derivation shows that the existence of these directions is independent of the cutoff function used.

$\dagger$ vipul@puhep1.princeton.edu 
In the Wilsonian[1] effective action approach to field theory, one works with an action that has a finite short distance cutoff. The idea is that if one changes this cutoff, one can find appropriate changes in the couplings of the action, so that physics much below the cutoff scale is invariant. This approach involves the presence of arbitrary interaction terms in the action, including those that in usual renormalizable quantum field theories would be regarded as non-renormalizable.

Halpern and Huang[2] studied the space of all possible non-derivative interaction terms about the Gaussian fixed point in $d$ dimensions, and found that there are non-polynomial interaction terms that are 'asymptotically free', i.e. as the short distance cutoff is taken to zero, these interactions also tend to zero. This is in contrast to polynomial potentials, which exhibit the opposite behaviour. This result is quite surprising, and may well have interesting physical implications, as mentioned by Halpern and Huang[2].

The Wegner-Houghton[3] implementation of the Wilson scheme was used in Ref. [2], so some care had to be taken to avoid pathologies associated with the use of a sharp cutoff. In the present note, I want to show that the result of Ref. [2] can be derived very simply from Polchinski's formulation[4] of the Wilson renormalization group. Questions of universality, i.e. independence from the manner of regularization, can also be addressed easily in this approach. The recursion relation derived in Ref. [2] is replaced here with a differential equation.

This note is organized as follows: I recall Polchinski's version of the Wilson renormalization group equation. I reduce this equation to the case needed for an analysis analogous to that of Ref. [2]. I show that this equation has the same solutions as the solutions found by other means in Ref. [2]. Next, I point out the physics behind these formal manipulations.

Start from a regulated partition function,

$$
\mathcal{Z}[J] \equiv \int \mathrm{D} \phi \mathcal{N}(a) \exp \left(-S_{a}[\phi]-\int J(p) \phi(-p)\right)
$$

where $\mathcal{N}(a)$ is a normalization factor given below. Divide $S_{a}=\int \frac{1}{2} \phi(x) \phi(y) a^{-2} K^{-1}((x-$ $\left.y)^{2} / a^{2}\right)+S_{a, i n t} \cdot a^{2} K\left(x^{2} / a^{2}\right)$ is the position-space representation of a cutoff Green function that differs from the inverse of the Laplacian only for large momenta. Let $\mathcal{N}(a) \equiv$ $\operatorname{det}^{-\frac{1}{2}}(K)$. We want $a \frac{\partial}{\partial a} \mathcal{Z}[J]=0$, for appropriate $J$, corresponding to long-wavelength correlation functions. Writing

$$
\begin{aligned}
\mathcal{Z}[J]=\exp \left(-S_{a, \text { int }}\left[-\frac{\delta}{\delta J}\right]\right) \int \mathrm{D} \phi \mathcal{N}(a) & \exp \left(-\int \frac{1}{2} \phi(x) \phi(y) a^{-2} K^{-1}\left((x-y)^{2} / a^{2}\right)\right) \\
& \times \exp \left(-\int J(x) \phi(x)\right),
\end{aligned}
$$


we find

$$
\mathcal{Z}[J]=\exp \left(-S_{a, i n t}\left[-\frac{\delta}{\delta J}\right]\right) \exp \left(\int \frac{1}{2} J(x) J(y) a^{2} K\left((x-y)^{2} / a^{2}\right)\right) .
$$

This rewriting of $\mathcal{Z}[J]$ is essentially algebraic.

Now acting with $a \frac{\partial}{\partial a}$ on $\mathcal{Z}[J]$, and performing some (functional) integrations-by-parts, we find

$a \frac{\mathrm{d} S_{a, i n t}}{\mathrm{~d} a}=\frac{1}{2} \int\left[\frac{\delta\left(S_{a, i n t}+\int J \phi\right)}{\delta \phi(x)} \frac{\delta\left(S_{a, i n t}+\int J \phi\right)}{\delta \phi(y)}-\frac{\delta^{2} S_{a, i n t}}{\delta \phi(x) \delta \phi(y)}\right] a \frac{\partial}{\partial a} a^{2} K\left((x-y)^{2} / a^{2}\right)$.

ensures that all correlation functions are left invariant. Two comments are in order here: (1) The integrations-by-parts, in this case, do not give any boundary terms, subject to the assumed validity of our algebraic treatment of $\mathcal{Z}[J] ;(2)$ For $J$ such that the Fourier transform of $J$ vanishes for large $p, J$ disappears from the above equation.

In general, $S_{a, \text { int }}$ must include arbitrary interaction terms, including derivative interactions. We are interested at present in examining the rg behaviour of various interaction terms in the vicinity of the Gaussian fixed point. In other words, we are looking at vectors in the tangent space to the space of all interactions, at the Gaussian fixed point, so we focus our attention on only a certain subspace of tangent vectors. Were we to try to follow the rg trajectory away from the fixed point, we would have to include all possible terms, as they show up in evolving $S_{a, i n t}$. If we consider $S_{a, i n t}=g \int a^{-d} U_{a}\left(\phi a^{d-2 / 2}\right)$, where $U_{a}$ is some function, for small $g$ we need only keep

$$
a \frac{\mathrm{d} S_{a, i n t}}{\mathrm{~d} a}=-\frac{1}{2} \int \frac{\delta^{2} S_{a, i n t}}{\delta \phi(x) \delta \phi(y)} a \frac{\partial}{\partial a} a^{2} K\left((x-y)^{2} / a^{2}\right) .
$$

In fact, since we are assuming that there are no derivatives in $S_{a, \text { int }}$, we have just

$$
a^{d} a \frac{\partial}{\partial a} a^{-d} U_{a}\left(\phi a^{d-2 / 2}\right)=-\frac{1}{2} \frac{\partial^{2}}{\partial \phi^{2}} U_{a}\left(\phi a^{d-2 / 2}\right) \times a^{d-2} a \frac{\partial}{\partial a} a^{2} K(0)
$$

Thus,

$$
a \frac{\partial}{\partial a} U_{a}(x)+\frac{d-2}{2} x U_{a}^{\prime}(x)-d U_{a}(x)=+\frac{1}{2} U_{a}^{\prime \prime}(x) \kappa .
$$

Here we have defined

$$
\kappa=-a^{d-2} a \frac{\partial}{\partial a} a^{2} K(0),
$$

which in momentum space is just

$$
\kappa=-a^{d} \int \frac{d^{d} p}{(2 \pi)^{d}} \frac{1}{a^{2}} a \frac{\partial}{\partial a} a^{2} \tilde{K}\left(p^{2} a^{2}\right) .
$$


We made no assumptions about the form of the cutoff function in this derivation, and all dependence on $K$ is through $\kappa=\kappa(K)$.

Now, suppose we look for functions $U_{a}$ that scale as functions of $a$, i.e. functions such that

$$
a \frac{\partial}{\partial a} U_{a}(x)=\lambda U_{a}(x)
$$

Then we want to solve

$$
(\lambda-d) f(x ; \lambda)-\frac{\kappa}{2} f^{\prime \prime}(x ; \lambda)+\frac{d-2}{2} x f^{\prime}(x ; \lambda)=0 .
$$

It is trivial to show that even solutions of this equation are exactly those found by Halpern and Huang, who imposed $f(0 ; \lambda)=0$. The only difference as compared to Ref. [2] is that we have found $\kappa$ for a general cutoff function, where they found the volume of a $d-1$ dimensional sphere divided by $(2 \pi)^{d}$. In fact, for a sharp cutoff, $\kappa$ takes exactly the value they found. The solutions are given in terms of the Kummer function:

$$
M(a, b, c) \equiv \frac{\Gamma(b)}{\Gamma(b-a) \Gamma(a)} \int_{0}^{1} d t \exp (t c) t^{a-1}(1-t)^{b-a-1}
$$

with

$$
f(x ; \lambda)=\left[M\left((\lambda-d) /(d-2), 1 / 2,(d-2) x^{2} / 2 \kappa\right)-1\right] .
$$

Various properties of these potentials are explained in Ref. [2], so I will not go into the details.

One should not suppose that perturbation theory for such interaction potentials is simple. The case of interest is $\lambda>0$, since for such eigenvalues, as $a \downarrow 0, U_{a}$ scales down. In fact, the full potential is

$$
V(\phi) \equiv g a^{\lambda-d} U_{1}\left(\phi a^{d-2 / 2}\right)
$$

Thus the 'anomalous' dependence on $a$ is as $a^{\lambda}$, which is much stronger than the logarithmic scale dependence one finds for dimensionless couplings in asymptotically free renormalizable field theories. $V(\phi)$ is to be treated in its entirety as a perturbation, in contrast to usual field theory where one rescales the interaction monomials so that higher order monomials are multiplied by higher powers of the coupling constant. What this implies is that even at the lowest order in perturbation theory one has to sum over an infinite set of graphs. For any $n$-point connected correlation function, at first order in perturbation theory the contributions are from graphs with a single vertex, but there are vertices of arbitrarily high degree, implying that most of the contractions are of tadpole type. A 
moment's reflection will make it evident that this is precisely what is encoded in the differential equation that determines these potentials. The scaling behaviour is dependent on the existence of such an infinite series of terms.

It is important to note, as well, that one cannot draw immmediate conclusions about the scaling behaviour of low energy correlation functions when their arguments are scaled from the behaviour of the coupling parameter in the action. In fact, low energy correlation functions are independent of $a$ by construction. One must compute to second order in the perturbation before one finds a nontrivial $\beta$ function with the same interpretation as the $\beta$ functions of renormalizable field theories - as mentioned above, the scaling found above comes from tadpole type graphs, and these have no dependence on external momenta at all.

One may wonder if the term quadratic in $S_{a, i n t}$ in the rg equation would change the scaling behaviour found in Ref. [2]. The term quadratic in $S_{a, \text { int }}$ could contribute to the evolution of the potential, but in fact it does not if the cutoff function satisfies

$$
\lim _{p \downarrow 0} \frac{\partial}{\partial a} a^{2} \tilde{K}\left(p^{2} a^{2}\right)=0 .
$$

This is a very reasonable restriction on cutoff functions, since it merely implies that at low momenta the propagator is independent of the cutoff. So the linear equation stays valid for the flow of the potential terms, except for the fact that the field needs to be rescaled at second order, and derivative interactions in $S_{a, i n t}$ can also contribute to the flow of the potential terms.

The effect of field rescaling is approximately of the form

$$
f(x ; \lambda)=g\left[M\left((\lambda-d) /(d-2+2 \eta), 1 / 2,(d-2+2 \eta) x^{2} / 2 \kappa\right)-1\right],
$$

except that $\eta=O\left(g^{2}\right)$ is not trivial to determine, and indeed depends on higher moments of the cutoff propagator. This is how the evolution of the kinetic term produces changes in the flow of the effective potential. The result of Halpern and Huang[2] is clearly unaffected by such nonlinear terms, since their calculation was expressly to determine the relevant directions in the space of perturbations of a scalar effective field theory. It would be extremely interesting to study the evolution of such relevant directions numerically.

This work was supported in part by NSF Grant No. PHY90-21984.

\section{References}

1. K. Wilson, in Collective properties of physical systems, (B. Lundqvist and S. Lundqvist, eds.), Academic Press, New York, 1973 K.G. Wilson and J.B. Kogut, Phys. Rep. 12C 
(1974) 75; F. Wegner, in Phase transitions and critical phenomena, vol. 6, (C. Domb and M. Green, eds.), Academic Press, New York, 1976

2. K. Halpern and K. Huang, Phys. Rev. Lett. 74 (1995) 3526; MIT report, CTP 2477 (1995)

3 F. Wegner and A. Houghton, Phys. Rev. A8 (1972) 401;

4 J. Polchinski, Nucl. Phys. B231 (1984) 269 